\title{
The Comparison of Computed Tomography Perfusion, Contrast-Enhanced Computed Tomography and Positron-Emission Tomography/Computed Tomography for the Detection of Primary Esophageal Carcinoma
}

\author{
Berhan Genc $^{a}$ b Mecit Kantarci $^{b}$ Recep Sade ${ }^{b}$ Ebru Orsal ${ }^{c}$ Hayri Ogul ${ }^{b}$ \\ Aylin Okur $^{\mathrm{e}}$ Yener Aydin $^{\mathrm{d}}$ Leyla Karacab $^{\mathrm{b}}$ Atilla Eroğlu $^{\mathrm{d}}$ \\ ${ }^{a}$ Department of Radiology, School of Medicine, Sifa University, Izmir, Departments of ${ }^{b}$ Radiology, \\ ${ }^{\mathrm{c} N u c l e a r}$ Medicine and ${ }^{\mathrm{d}}$ Thoracic Surgery, School of Medicine, Ataturk University, Erzurum, and \\ eDepartment of Radiology, School of Medicine, Bozok University, Yozgat, Turkey
}

\section{Key Words}

Computed tomography · Esophageal cancer · Computed tomography perfusion · Positron-emission tomography

\footnotetext{
Abstract

Objective: The purpose of this study was to investigate the efficiency of computed tomography perfusion (CTP), contrast-enhanced computed tomography (CECT) and ${ }^{18} \mathrm{~F}$-fluoro-2-deoxy-D-glucose $\left({ }^{18} \mathrm{~F}-\mathrm{FDG}\right)$ positron-emission tomography (PET/CT) in the diagnosis of esophageal cancer. Subjects and Methods: This prospective study consisted of 33 patients with pathologically confirmed esophageal cancer, 2 of whom had an esophageal abscess. All the patients underwent CTP, CECT and PET/CT imaging and the imaging findings were evaluated. Sensitivity, specificity and positive and negative predictive values were calculated for each of the 3 imaging modalities relative to the histological diagnosis. Results: Thirty-three tumors were visualized on CTP, 29 on CECT and 27 on $\mathrm{PET} / \mathrm{CT}$. Six tumors were stage 1 , and 2 and 4 of these tumors
}

\begin{tabular}{ll}
\hline KARGER & $\begin{array}{l}\text { ( ) 2016 S. Karger AG, Basel } \\
1011-7571 / 16 / 0253-0254 \$ 39.50 / 0\end{array}$ \\
$\begin{array}{l}\text { E-Mail karger@karger.com } \\
\text { www.karger.com/mpp }\end{array}$ & $\begin{array}{l}\text { This is an Open Access article licensed under the terms of the } \\
\text { Creative Commons Attribution-NonCommercial 3.0 Un- } \\
\text { ported license (CC BY-NC) (www.karger.com/OA-license), } \\
\text { applicable to the online version of the article only. Distribu- } \\
\text { tion permitted for non-commercial purposes only. }\end{array}$
\end{tabular}

were missed on CECT and PET/CT, respectively. Significant differences between CTP and CECT ( $p=0.02)$, and between CTP and PET/CT $(p=0.04)$ were found for stage 1 tumors. Values for the sensitivity, specificity and positive and negative predictive values on CTP were 100,100, 100 and $100 \%$, respectively. Corresponding values on CECT were $93.94,0,93.94$ and $0 \%$, respectively, and those on PET/CT were $87.88,0,93.55$ and $0 \%$, respectively. Hence, the sensitivity, specificity and positive and negative predictive values of CTP were better than those of CECT and PET/CT. Conclusion: CTP had an advantage over CECT and PET/CT in detecting small lesions. CTP was valuable, especially in detecting stage 1 tumors.

c) 2016 S. Karger AG, Basel

\section{Introduction}

Esophageal cancer is the third-most common gastrointestinal malignancy and has one of the worst prognoses of all malignancies [1]. Squamous-cell carcinoma and 
adenocarcinoma together constitute $>90 \%$ of all esophageal cancers [2]. Treatment options, depending on the disease stage, include surgery, chemotherapy, radiotherapy or multimodality therapy. Surgical resection is currently the best curative treatment in cases with no distant metastases or with locally advanced tumor growth [3]. Thus, early diagnosis is of utmost importance. Like many malignancies, the staging of esophageal cancer incorporates information regarding the depth of local invasion, regional lymph node involvement and the presence of distant metastases. Correct staging before therapy is of paramount importance for selecting the appropriate therapeutic modality [3-5]. For correct diagnosis and staging, diagnostic modalities such as contrast-enhanced computed tomography (CECT), endoscopic ultrasonography (EUS) and ${ }^{18} \mathrm{~F}$-fluoro-2-deoxy-D-glucose $\left({ }^{18} \mathrm{~F}\right.$ FDG) positron emission tomography with computed tomography (PET/CT) are used in combination. The CECT scan effectively localizes the primary tumor, determines the extent of involvement and also whether or not an invasion of adjacent structures is present. PET/CT evaluates high glucose metabolism in tumor tissue compared with normal tissue and enables a quantitative assessment of the tumor's metabolic activity, i.e. maximum standardized uptake value $\left(\mathrm{SUV}_{\max }\right)$, in addition to a visual assessment of the primary and metastatic tumors. FDG-PET/CT offers the unique combination of functional PET and anatomic CT imaging, which facilitates the identification of primary and secondary tumors [6]. It has also been used for assessing response to therapy. However, determination of the small tumors in the esophagus is not efficient enough with conventional imaging modalities $[3,4,7]$. Several studies have reported normal PET/CT scans despite the presence of primary esophageal cancer $[3-5,8]$.

Computed tomography perfusion (CTP) imaging has been used increasingly in oncological practice [9-11]. With CTP, it is possible to evaluate the development of abnormal new vessels (angiogenesis) in tumor tissues in a noninvasive and quantitative manner [9]. Tumor angiogenesis is an important mechanism in tumor growth and spread. Hence, a high vascularity of the tumor tissue indicates aggressive tumor behavior and a worse prognosis. It is also possible with CTP to accomplish a quantitative assessment using parameters such as the blood volume (BV), blood flow (BF) and permeability (PMB) in tumors [1]. Hence, the aim of this study was to evaluate the efficiency of CTP, CECT and PET/CT in the diagnosis of esophageal cancer.

CTP Imaging in Esophageal Cancer

\section{Subjects and Methods}

\section{Patients}

Thirty-three patients with histopathologically confirmed esophageal cancer and 2 patients with nonneoplasia were included. This study was approved by the Institutional Ethical Scientific Committee and based on the Declaration of Helsinki. Written informed consent was obtained from each participant after explaining the purpose of this study. All examinations were performed within a 7-day interval. Two patients were diagnosed with esophageal abscess from biopsy specimens. The standard of reference for diagnosis of the esophageal carcinoma was the histopathological finding on a biopsy specimen obtained by endoscopy. Twenty-five patients were diagnosed with squamous-cell carcinoma and 8 with adenocarcinoma from biopsy specimens. Of the 33 patients, 6 had stage 1 tumors, 5 had stage 2 tumors, 10 had stage 3 tumors and 12 had stage 4 tumors. The tumor diameter was $5-15 \mathrm{~mm}$ in 12 patients, $15-25 \mathrm{~mm}$ in 9 patients and $25-35 \mathrm{~mm}$ in 12 patients.

\section{CTP Technique}

All CTP examinations were performed using a 2nd-generation Somatom Definition Flash 256-slice dual-source MDCT scanner (Siemens Healthcare, Forchheim, Germany) with the following protocol. The scanning region was restricted to the length of the tumor based on the EUS finding. The patients were required to lie still on the table throughout the rapid injection of the iodinated contrast material, $50 \mathrm{ml}$ of iopromide (Ultravist $370 \mathrm{mg} / \mathrm{ml}$, Bayer Schering Pharma, Berlin, Germany). An automatic injector was used (MCT Plus; Medrad, Pittsburgh, Pa., USA) over $12.5 \mathrm{~s}$ through an 18-gauge intravenous line placed into the right antecubital vein at a rate of $4 \mathrm{ml} / \mathrm{s}$. The contrast produced a sensation of 'hot flash'. Immediately following the injection of the iodinated contrast, $50 \mathrm{ml}$ of saline was infused by the same injector via the same route. After $6 \mathrm{~s}$, multiple scans with a prespecified scan range were obtained using the 4-dimensional spiral-mode with the following device settings: a tube potential of $80 \mathrm{kVp}$, an effective tube current of $100 \mathrm{mAs} / \mathrm{rot}$, a scan quantity of 25, an alternating scan direction, a table travel time from one end point to the other of $1.5 \mathrm{~s}$, a total examination time of $39 \mathrm{~s}$, a slice acquisition of $128 \times$ $0.6 \mathrm{~mm}$ (using the $\mathrm{z}$-flying spot) and a Gantry rotation time of $0.28 \mathrm{~s}$. The perfusion scan phase required the patients to respire as shallowly and slowly as possible. The image reconstruction was conducted with a slice thickness of $3 \mathrm{~mm}$ and increments of $3 \mathrm{~mm}$; in addition, a medium-smooth tissue convolution kernel (B20f) was utilized. For further analyses, the reconstructed images were transferred to a work station (Leonardo, Siemens Medical Solutions).

The dose reduction technique was applied. The mean radiation volume CT dose index and dose-length product (DLP) of CTP scans were recorded for each patient. The effective radiation dose of CTP was calculated by applying a method proposed by the European Working Group guideline on quality criteria for CT, which uses the DLP and a conversion coefficient for the thorax, i.e. $\mathrm{k}=$ $0.015 \mathrm{mSv} /(\mathrm{mGy} \mathrm{cm})$.

The CTP data were processed on a special work station (Leonardo Volume Perfusion CT Body, Siemens). The perfusion was calculated using the maximum-slope method. To obtain the reference arterial input information, the region of interest (ROI) was placed on the descending aorta at the level of the diaphragm, and the ROI (range $15-32 \mathrm{~mm}^{2}$ ) was drawn manually. The arterial time-attenuation curve was automatically drawn, and this arterial

Med Princ Pract 2016;25:254-259 

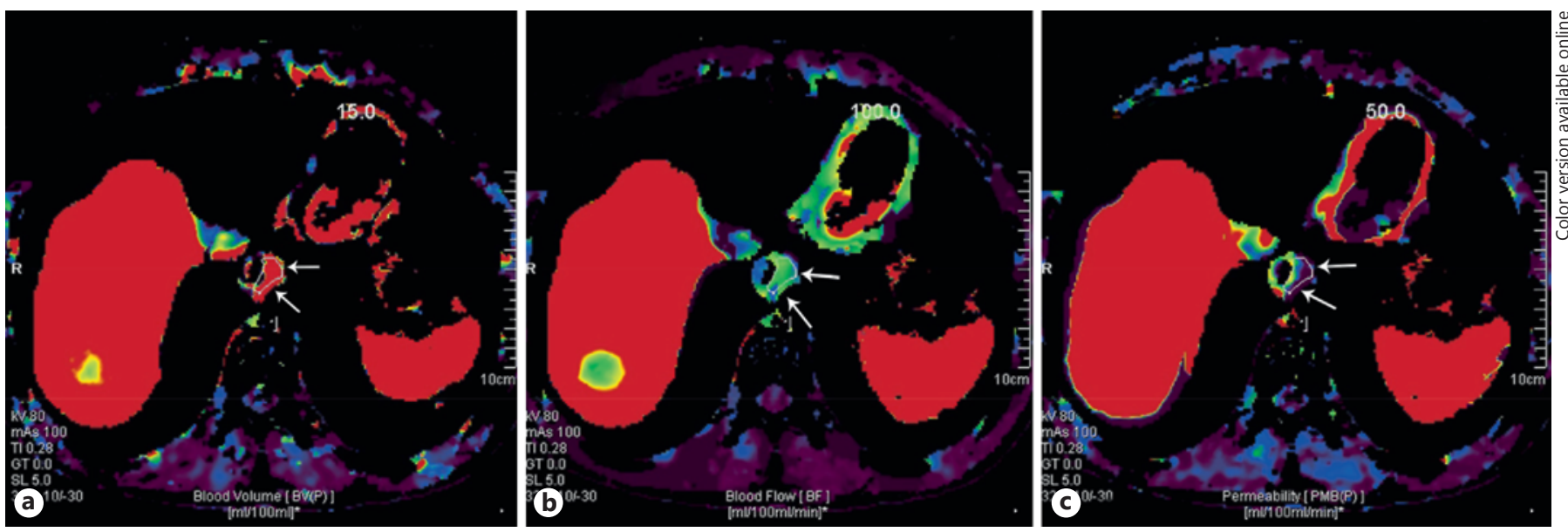

Fig. 1. a-c A 56-year-old male patient with a 4-mm distal esophageal mass, with biopsy-proven squamous-cell carcinoma. On CTP, color maps of BV (a), BF (b) and PMB (c) reveal the intratumoral perfusion distribution of mass (arrows).

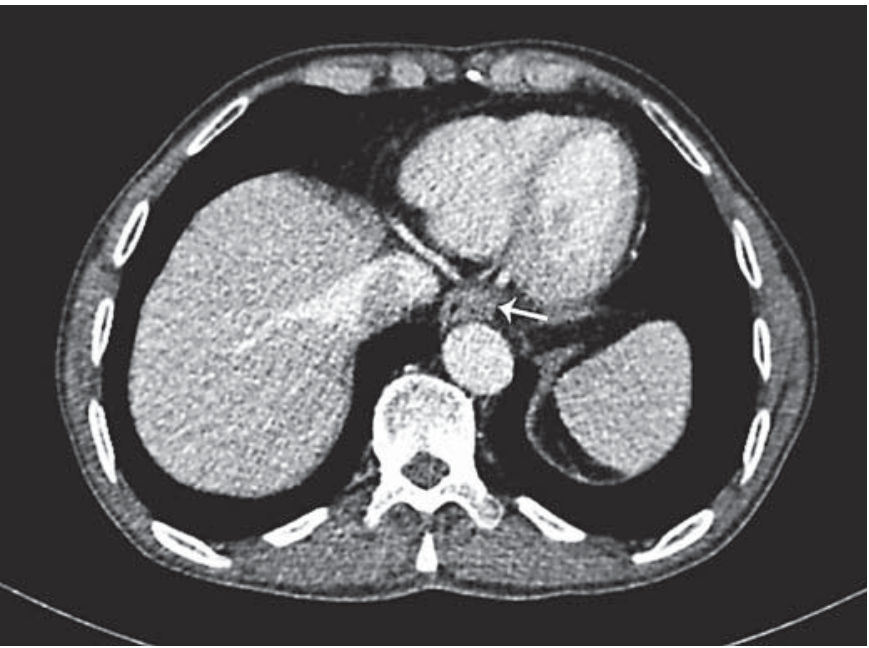

Fig. 2. A 56-year-old male patient with a 4 -mm distal esophageal mass, with biopsy-proven squamous-cell carcinoma. Axial CECT image shows an esophageal mass (arrows) that did not enhance at the same level.

input information was recorded by the software for future calculations. The functional maps had a color range from purple to red, with the former indicating the lowest border and the latter indicating the uppermost border of the display for the BF (color range $0-100), \mathrm{BV}$ (color range $0-15$ ) and PMB (color range $0-50)$. The ROIs (range $55-160 \mathrm{~mm}^{2}$ ) were manually drawn for each tumor. To draw the ROI from an area within the tumor, the area with maximal contrast uptake was selected in an attempt to avoid the surrounding tissues and vascular structures. BF (ml/100 g tissue/ $\mathrm{min}), \mathrm{BV}(\mathrm{ml} / 100 \mathrm{~g}$ tissue) and PMB ( $\mathrm{ml} / 100 \mathrm{ml} / \mathrm{min})$ were calculated using the ROIs drawn from the tumor. The diagnosis was based on the visual analysis of the colored parametric CTP maps. Analysis of the images was performed by 2 radiologists (M.K. with
10 years of experience in thoracoabdominal radiology and B.G. with 8 years of experience in radiology). They reviewed the images in consensus to ensure the accuracy of the quantitative analyses.

\section{CECT Technique}

All CECT examinations were performed using the same scanner that was used for CTP as well as the same contrast medium and contrast injection technique. Thirty seconds after the start of the injection, scans with a prespecified scan range (thorax) were done using the 4-dimensional spiral-mode with the following device settings: a tube potential of $100 \mathrm{kVp}$, an effective tube current of 120 $\mathrm{mAs} / \mathrm{rot}$, a scan quantity of 25 , an alternating scan direction, a table travel time from one end point to the other of $1.5 \mathrm{~s}$, a total examination time of $5 \mathrm{~s}$, a slice acquisition of $128 \times 0.6 \mathrm{~mm}$ (using the $\mathrm{z}$-flying spot) and a Gantry rotation time of $0.28 \mathrm{~s}$. The image reconstruction was performed using a slice thickness of $1 \mathrm{~mm}$, in addition to using a medium-smooth tissue convolution kernel (B20f). For further analyses, the reconstructed images were transferred to a work station (Leonardo).

The criteria included in diagnosing the lesion were: length of the involved segment, dilated fluid-filled and debris-filled esophageal lumen proximal to an obstructing lesion, symmetric or asymmetric wall thickening $(>5 \mathrm{~mm}$ ), homogeneous or heterogeneous enhancement and associated findings like soft-tissue mass and lymph nodal and distant metastases. Analysis of the images was performed by M.K. and B.G. reviewing the images in consensus.

\section{FDG-PET/CT Imaging Technique}

All studies were performed with PET/CT equipment (Siemens, Knoxville, Tenn., USA) consisting of a PET scanner coupled to an 8-detector-row CT scanner, allowing for the simultaneous acquisition of coregistered PET and CT images. According to the study protocol, ${ }^{18} \mathrm{~F}-\mathrm{FDG}$ of $444-555 \mathrm{MBq}(12-15 \mathrm{mCi})$ was administered intravenously after at least $6 \mathrm{~h}$ of fasting. Blood glucose levels were checked before the tracer injection. Whole-body PET/CT acquisition was begun approximately $1 \mathrm{~h}$ after the tracer injection. A low-dose CT scan was taken from the vertex to the thigh, and then PET images were taken in the supine position as $7-9$ beds ( $3 \mathrm{~min} /$ 

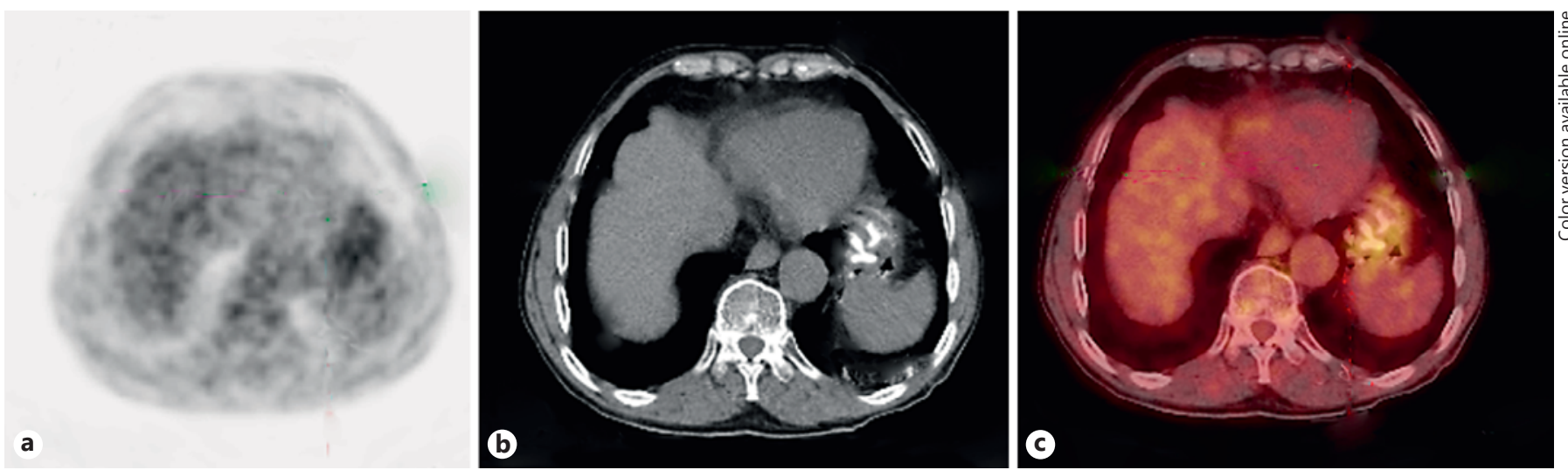

Fig. 3. a-c A 56-year-old male patient with a 4-mm distal esophageal mass, with biopsy-proven squamous-cell carcinoma. On the axial PET image (a), CT image (b) and axial fused PET/CT image (c), the primary tumor demonstrates no significant FDG uptake.

bed). The CT and PET images were fused into transaxial, coronal and sagittal sections. Higher uptake of ${ }^{18}$ F-FDG compared with the background activity was interpreted as abnormal, and CT images were used for anatomic landmarking. The ROI was drawn manually from the image that showed the most intense ${ }^{18} \mathrm{~F}-\mathrm{FDG}$ uptake in the primary esophageal lesion, and the $\mathrm{SUV}_{\max }$ was calculated automatically.

The PET, CT and fused PET/CT images were transferred to a Leonardo work station. The images were examined by a nuclearmedicine physician (E.O., who had specialized clinical knowledge regarding esophageal cancer and 5 years of experience with PET/ CT). Areas that displayed a higher ${ }^{18}$ F-FDG uptake on PET/CT were considered positive for esophageal cancer.

\section{Statistical Analysis}

The statistical analyses were performed with a commercially available software package (Statistical Package for Social Sciences, v20.0, SPSS Inc., Chicago, Ill., USA). The data were grouped by pathological cell type and lesion stage. The pathological groups consisted of neoplasia and nonneoplasia. The lesion stage groups consisted of stages 1-4. They were compared across groups using the Kruskal-Wallis rank-sum test. A patient's value was calculated for each comparison and $\mathrm{p}<0.05$ was accepted as statistically significant. Sensitivity, specificity and positive and negative predictive values were calculated for CTP, CECT and PET/CT relative to the histological diagnosis.

\section{Results}

The mean age of the patients was $58 \pm 14.8$ years. All the patients tolerated CTP, CECT and PET/CT examinations well. No problems occurred during or at the end of the procedures. The mean radiation volume CT dose index was $88.65 \mathrm{mGy}$. The calculated effective radiation dose for the perfusion scans was 32.9 [DLP $(2,193 \mathrm{mGy} \times \mathrm{cm}) \times \mathrm{k}$ $(0.015 \mathrm{mSv} / \mathrm{mGy} \times \mathrm{cm})] \mathrm{mSv}$. Six patients were found to have distant metastasis and 11 had lymph node metastases on whole-body PET/CT.

All tumors were seen on CTP. Two were not seen on PET/CT (fig. 1-3) and another 2 were not seen on CECT or PET/CT; these were all stage 1 tumors. Hence, CTP was more effective in detecting stage 1 tumors than CECT and PET/CT (table 1). The 2 abscesses were misdiagnosed as tumors on CECT and PET/CT. Abscesses and tumors were distinguished using CTP (table 2), but CECT and PET/CT did not distinguish between them. Sensitivity, specifity and positive and negative predictive values on CTP were better than on CECT and PET/CT (table 2). The perfusion and $S U V_{\max }$ values are in table 3.

\section{Discussion}

In this study, CTP visualized all the tumors, CECT missed 2 and PET/CT missed 4 of the stage 1 esophageal cancer tumors. CTP also had advantages over CECT and $\mathrm{PET} / \mathrm{CT}$ in the differentiation of neoplasia and nonneoplasia.

PET/CT has certain limitations in the diagnosis of primary esophageal cancer $[3,5]$. The probable explanation for PET/CT missing 4 tumors was that, in the gastroesophageal region, inflamed gastric mucosa could show as marked FDG uptake, thereby making differentiation between an inflammatory lesion and a tumor difficult. However, cases without apparent FDG uptake on PET/ CT were reported despite the presence of esophageal cancer $[5,9,12]$. Due to limited spatial resolution, PET/CT could result in false-negatives in the setting of small tumors. Flamen et al. [5] found false-negative PET images 
Table 1. Comparison of CTP, CECT and PET/CT

\begin{tabular}{|c|c|c|c|c|c|c|c|c|}
\hline & \multirow{2}{*}{$\begin{array}{l}\text { Endoscopic } \\
\text { pathology }\end{array}$} & \multicolumn{2}{|l|}{ CTP } & \multicolumn{2}{|l|}{ CECT } & \multicolumn{2}{|l|}{$\mathrm{PET} / \mathrm{CT}$} & \multirow[t]{2}{*}{$\mathrm{p}$ value } \\
\hline & & positive & negative & positive & negative & positive & negative & \\
\hline Stage 1 & 6 & 6 & 0 & 4 & 2 & 2 & 4 & $<0.05^{*}, \#$ \\
\hline Stage 2 & 5 & 5 & 0 & 5 & 0 & 5 & 0 & $>0.05^{*, \#}$ \\
\hline Stage 3 & 10 & 10 & 0 & 10 & 0 & 10 & 0 & $>0.05^{*, \#}$ \\
\hline Stage 4 & 12 & 12 & 0 & 12 & 0 & 12 & 0 & $>0.05^{*, \#}$ \\
\hline Total & 33 & 31 & 2 & 33 & 0 & 29 & 4 & $>0.05^{*},<0.05^{\#}$ \\
\hline
\end{tabular}

* CTP versus CECT; ${ }^{*}$ CTP versus PET/CT.

Table 2. Sensitivity, specifity and positive and negative predictive values of CTP, CECT and PET/CT

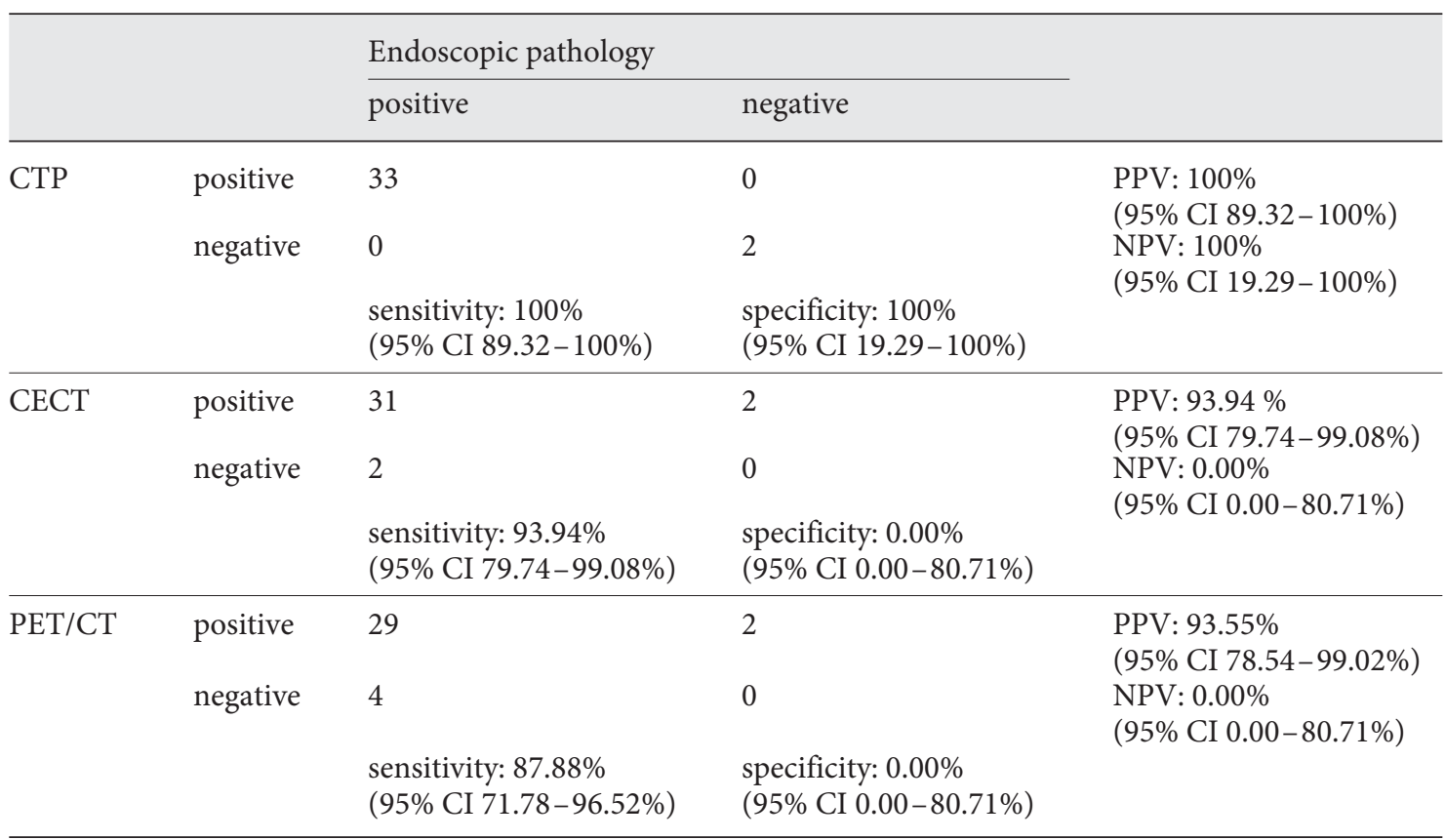

$\mathrm{CI}=$ Confidence interval; NPV = negative predictive value; $\mathrm{PPV}=$ positive predictive value

Table 3. Comparison of the tumor stage, CTP parameters and $S U V_{\max }$ values calculated in the tumor

\begin{tabular}{|c|c|c|c|c|c|}
\hline Parameter & $\begin{array}{l}\text { Stage 1 } \\
(\mathrm{n}=6)\end{array}$ & $\begin{array}{l}\text { Stage } 2 \\
(\mathrm{n}=5)\end{array}$ & $\begin{array}{l}\text { Stage } 3 \\
(\mathrm{n}=10)\end{array}$ & $\begin{array}{l}\text { Stage } 4 \\
(\mathrm{n}=12)\end{array}$ & $\mathrm{p}$ value \\
\hline $\mathrm{BV}, \mathrm{ml} / 100 \mathrm{~g}$ & $7.52 \pm 3.65$ & $7.84 \pm 2.08$ & $8.86 \pm 2.19$ & $9.80 \pm 0.90$ & $>0.05^{*}$ \\
\hline $\mathrm{BF}, \mathrm{ml} / 100 \mathrm{~g} / \mathrm{min}$ & $86.56 \pm 90.78$ & $79.62 \pm 40.69$ & $87.55 \pm 39.33$ & $102.34 \pm 45.10$ & $0.021^{*}$ \\
\hline $\mathrm{PMB}, \mathrm{ml} / 100 \mathrm{ml} / \mathrm{min}$ & $14.09 \pm 9.88$ & $13.02 \pm 4.70$ & $12.62 \pm 3.22$ & $14.37 \pm 9.56$ & $>0.05^{*}$ \\
\hline $\mathrm{SUV}_{\max }$ & $9.2 \pm 6$ & $8.5 \pm 0.91$ & $7.3 \pm 6.4$ & $10.4 \pm 5.5$ & $>0.05$ \\
\hline
\end{tabular}

Data are presented as means \pm standard deviation. ${ }^{*}$ Adjusment for multiple comparisons: the Bonferroni correction. 
in esophageal cancers with a diameter $<8 \mathrm{~mm}$. On the other hand, CTP, due to its high-spatial-resolution power, enables the assessment of small lesions.

The CTP technique uses various perfusion parameters such as BF, BV, mean transit time and PMB, to demonstrate angiogenesis in tumor tissues and to noninvasively and quantitatively assess microcircular changes. Studies regarding the use of CTP in esophageal cancer have recently been published $[9,13-15]$. Several studies have reported that BV might be a good indicator of angiogenesis of esophageal tumors [3,13], and 1 study revealed that BF is a significant prognostic factor in esophageal tumors. It was also shown that low BF tumors were frequently inoperable [15]. Among various clinicopathological factors and CTP parameters, the significant relationship was that tumors with hematogenic metastasis exhibited a higher $\mathrm{BF}$ than those without it $[16,17]$.

Compared with PET/CT, our results indicate that CTP is feasible and provides useful information about small esophageal tumors. Furthermore, a negative CECT and $\mathrm{PET} / \mathrm{CT}$ in primary esophageal cancer cannot rule out an esophageal malignancy.

Our study has certain limitations. First, it had a small sample size, limiting its statistical power. Larger patient series with a wider range of patient groups are clearly necessary. Another limitation is that CTP involves a relatively high dose of radiation exposure. However, as in our case, dose reduction protocols may be used to limit exposure. Finally, only patients with malignancy and abscesses were included in the study. Its lack of a patient group consisting of benign conditions (leiomyoma, esophagitis, etc.) is another limitation.

\section{Conclusion}

All tumors including stage 1 tumors were visualized on CTP, but the stage 1 tumors were not detectable on PET/ $\mathrm{CT}$ and CECT. Hence, CTP could be considered valuable for the diagnosis of esophageal cancers, especially stage 1 tumors. It could also differentiate neoplasia from nonneoplasia. In the presence of any clinical suspicion, when CECT and PET/CT findings are normal, CTP imaging should be done.

\section{Disclosure Statement}

There are no conflicts of interest.

\section{References}

1 Urschel JD, Vasan H: A meta-analysis of randomized controlled trials that compared neoadjuvant chemoradiation and surgery to surgery alone for resectable esophageal cancer. Am J Surg 2003; 185:538-543.

2 Daly JM, Fry WA, Little AG, et al: Esophageal cancer: results of an American College of Surgeons patient care evaluation study. J Am Coll Surg 2000;190:562-572.

3 Kim TJ, Kim HY, Lee KW, et al: Multimodality assessment of esophageal cancer: preoperative staging and monitoring of response to therapy. Radiographics 2009;29:403-421.

4 Bruzzi JF, Munden RF, Truong MT, et al: $\mathrm{PET} / \mathrm{CT}$ of esophageal cancer: its role in clinical management. Radiographics 2007;27: $1635-1652$.

5 Flamen P, Lerut A, Van Cutsem E, et al: Utility of positron emission tomography for the staging of patients with potentially operable esophageal carcinoma. J Clin Oncol 2000;18: 3202-3210.

6 Pauwels EK, Coumou AW, Kostkiewicz M, et al: [18F]Fluoro-2-deoxy-D-glucose positron emission tomography/computed tomography imaging in oncology: initial staging and evaluation of cancer therapy. Med Princ Pract 2013;22:427-437.

7 Godoy MC, Bruzzi JF, Viswanathan C, et al: Multimodality imaging evaluation of esophageal cancer: staging, therapy assessment, and complications. Abdom Imaging 2013;38: 974-993.

>8 Karaosmanoğlu AD, Blake MA: Applications of PET-CT in patients with esophageal cancer. Diagn Interv Radiol 2012;18:171-182.

$\$ 9$ Kambadakone AR, Sahani DV: Body perfusion CT: technique, clinical applications, and advances. Radiol Clin North Am 2009;47: 161-178.

10 Djuric-Stefanovic A, Saranovic D, Masulovic $\mathrm{D}$, et al: Comparison between the deconvolution and maximum slope 64-MDCT perfusion analysis of the esophageal cancer: Is conversion possible? Eur J Radiol 2013;82:17161723.

11 Umeoka S, Koyama T, Togashi K, et al: Esophageal cancer: evaluation with triplephase dynamic CT initial experience. Radiology 2006;239:777-783.

12 Wallace MB, Nietert PJ, Earle C, et al: An analysis of multiple staging management strategies for carcinoma of the esophagus: computed tomography, endoscopic ultrasound, positron emission tomography, and thoracoscopy/laparoscopy. Ann Thorac Surg 2002;74:1026-1032.

13 Chen TW, Yang ZG, Li Y, et al: Quantitative assessment of first-pass perfusion of esophageal squamous cell carcinoma using 64-section MDCT: initial observation. Clin Radiol 2009;64:38-45.

14 Hagano K, Okazumi S, Shuto K, et al: Perfusion CT can predict the response to chemoradiation therapy and survival in esophageal squamous cell carcinoma: initial clinical results. Oncol Rep 2007;18:901-908.

15 Makari Y, Yasuda T, Doki Y, et al: Correlation between tumor blood flow assessed by perfusion CT and effect of neoadjuvant therapy in advanced esophageal cancers. J Surg Oncol 2007;96:220-229.

16 Hasan J, Byers R, Jayson GC: Intra-tumoural microvessel density in human solid tumours. Br J Cancer 2002;86:1566-1577.

17 Maehara Y, Kabashima A, Koga T, et al: Vascular invasion and potential for tumor angiogenesis and metastasis in gastric carcinoma. Surgery 2000;128:408-416. 